University of Nebraska - Lincoln

DigitalCommons@University of Nebraska - Lincoln

Robert Katz Publications

Research Papers in Physics and Astronomy

September 1989

Particle Tracks in Diverse Media

Robert Katz

University of Nebraska-Lincoln, rkatz2@unl.edu

Follow this and additional works at: https://digitalcommons.unl.edu/physicskatz

Part of the Physics Commons

Katz, Robert, "Particle Tracks in Diverse Media" (1989). Robert Katz Publications. 29.

https://digitalcommons.unl.edu/physicskatz/29

This Article is brought to you for free and open access by the Research Papers in Physics and Astronomy at DigitalCommons@University of Nebraska - Lincoln. It has been accepted for inclusion in Robert Katz Publications by an authorized administrator of DigitalCommons@University of Nebraska - Lincoln. 
Published in Nuclear Instruments and Methods in Physics Research B 40/41 (1989), pp. 1271-1274.

Copyright (C) Elsevier Science Publishers B.V. Used by permission. http://www.sciencedirect.com/science/journal/o168583X

\title{
Particle Tracks in Diverse Media
}

\author{
Robert Katz
}

University of Nebraska-Lincoln, Lincoln, NE 68588-O111, USA

When energetic heavy ions pass into a medium, they create a trail of excitations, ionizations, and secondary electrons whose effect is represented through their average radial "dose" distribution. The structure of the track depends on this and on the observed "end point." Tracks may be observed microscopically or through the gross effect of a particle beam. We take the medium to be an assemblage of "targets" whose response to gamma rays is approximated by the cumulative Poisson distribution. While most detectors are 1-hit systems, we have discovered 2-hit and up to 8-hit response. Folding the gamma-ray response into the radial dose distribution we obtain the probability for activating the target as a function of radial distance which is integrated to yield the action cross section, through which the effect of a beam may be calculated.

\section{Introduction}

In the physics of particle track structure, detectors are assumed to consist of an assemblage of identical targets, whose response to ionizing radiations may be calibrated by exposing the detector to gamma rays.

As models of this response we use formulations from biological target theory called the multihit and multi-target models. For most physical detectors we use the multihit model based on a cumulative Poisson distribution. Here $C$ is the minimum number of hits per target needed to activate it, $A$ the number of trials per target and $X$ the number of hits per target. We have the probability that, after $A$ trials, a target will experience $X$ hits as

$$
P(X, A)=A^{X} \mathrm{e}^{-A} / X !,
$$

and the probability that a target will experience $C$ or more hits as

$$
P(C, \mathrm{~A})=1-\sum_{X=0}^{X=C-1} P(X, A) .
$$

We typically use a multitarget model for biological cells, giving the probability that $m$ targets will each experience 1 or more hits as

$$
P(m, A)=\left(1-\mathrm{e}^{-A}\right)^{m} .
$$

In these expressions $A$, the average number of trials per target, is taken to be the ratio of the dose of gamma rays, $E$, to the dose at which there is an average of 1 hit per target, $\mathrm{E}_{\mathrm{O}}$. That is,

$$
A=E / E_{\mathrm{o}}
$$

Most physical and some biological systems are 1-hit in their response to gamma rays. We have found values of $C$ as high as 8 in desensitized nuclear emulsions and $m$ as high as 6 in radiobiology.

In this model of particle tracks [1] each detector is represented by experimental parameters, with no attempt to analyze the mechanism. The model for physical detectors utilizes 3 parameters: $E_{0}$, the dose at which there is an average of 1 hit per target, $C$, the hittedness, and $a_{\mathrm{o}}$ the target size. Biological cells have greater structural complexity, for the sensitive elements and their subtargets are found within a cell nucleus [2]. For a description of cellular response we require a fourth parameter, $\sigma_{0}$, which may approximate the cross-sectional area of the cell nucleus, and we find it convenient to use as other parameters $m, E_{0}$, and $\kappa$, the latter being a combination of $E_{\mathrm{o}}$ and $a_{\mathrm{o}}$ [see Equation (8)].

\section{Nuclear emulsions as prototype detectors}

Our view of particle tracks is based on observed tracks of heavy ions and electrons in nuclear emulsions [3]. Here the track of a fast electron or proton often appears as a string of isolated developed grains. One measures the number of grains per unit path length along the presumed path of a particle. If $N$ is the number of undeveloped grains per unit volume, and $\sigma$ is the action cross section for producing a developable grain, then the number of developed grains per unit pathlength is $\sigma N$. If we wish to calculate the number of developed grains per unit energy deposition in an isolated track, we divide this by the stopping power $L$ to get a $G$-value (most frequently used in radiation chemistry) as

$$
G=\sigma N / L
$$

We refer to tracks which look like a collection of ran- 
domly spaced beads on a string as being in the "grain count" regime. For such tracks the energy deposition within several target radii of the ion's path plays a central role. Here the size of the target is significant, and we must average the radial dose over the target volume to estimate the response. The action cross section is typically smaller than the cross-sectional area of the target.

Where there are no obvious grains, the "grain size" may be an approximation to some characteristic distance, like a diffusion length. Such an approximation has been made for heavy ion radiolysis in water and benzene, and for scintillation counters, thermoluminescent dosimeters and other solid-state detectors. In these detectors there may be different response characteristics for different end points. The production of $\mathrm{HO}_{2} \bullet$ radicals in water is a 2-hit process as is the production of $\mathrm{H}_{2}$ from benzene. In our analysis of these processes we have taken $N$ to be the reciprocal of the target volume, and have used Equation (5) to calculate $G$-values [4]. In the Fricke dosimeter, the creation of $\mathrm{Fe}^{3+}$ from $\mathrm{Fe}^{2+}$ ions by the products of water radiolysis is a 1-hit process [5]. Thus, the same substance, liquid water, can behave like a 2-hit or a 1-hit detector depending on the observed end point.

With heavy ions the track in emulsion often looks like a hairy rope, as large numbers of energetic secondary electrons are produced, some of which can penetrate to considerable radial distance from the ion's path, perhaps to hundreds of grain diameters. We refer to such tracks as being in the "track width" regime. In such a track the probability of making a grain developable is virtually 1 , close to the ion's path. Here the precise knowledge of the radial dose close to the ion's path and knowledge of the target size is less significant than in the grain count regime. Calculation of the cross section may be made in a "point target" approximation. The cross section here bears much less relation to the target size than to the radial distance at which the dose approximates $E_{0}$. The action cross section may be several orders of magnitude larger than the actual target area.

With heavy-ion tracks the appearance of the track depends markedly on hittedness. As an oversimplification suppose that the hittedness refers to the number of electrons which must pass through a grain to render it developable. When $C=1$, grains will be developed to the outermost reach of delta ray penetration. At higher $C$ electron tracks may be unobservable, and the track of a heavy ion is observable only where the density of delta rays is sufficiently high that $C$ electrons are likely to pass through a grain. One might then speak of a "track core" effect, for there are always many more low-energy delta rays of limited radial penetration than there are highenergy delta rays. The phrase "track core effects," or the analogous concept of a "thermal spike," has been used as a qualitative description of events taking place close to the ion's path. We know of no valid evidence for either a track core in energy deposition or of a thermal spike. In radiobiology a question is raised about an apparently infinite relative biological effectiveness (RBE) of heavy ions in sister chromatid exchanges [6]. We suggest that this is a 2-hit process in which a single electron cannot initiate the observed effect. Moderate doses of Xrays will yield no observable effect, while a single heavy ion does.

When dealing with the track width regime in a nuclear emulsion, the innermost part of the track is nearly opaque. The probability for grain activation, $P$, is nearly 1 close to the ion's path, where many electrons may pass through a grain, and takes some lesser value at larger distances, down to o beyond the maximal radial distance, $T$, to which secondary electrons can penetrate, corresponding to a kinematic energy limit. The distance $T$ is partially determined by the particle speed $\beta_{c}$, where $c$ is the speed of light. Since the action cross section is found as the radial integral of the activation probability, $P$, we have

$$
\sigma=2 \pi \int_{t=0}^{t=T} P \mathrm{~d} t,
$$

which can be considerably less (in the grain count regime) or very much larger (in the track width regime) than the cross-sectional area of a grain. In the inactivation of dry enzymes and viruses by energetic heavy ions the inactivation cross section is found to be orders of magnitude larger than the cross-sectional area of the enzyme or virus molecule [7].

The greatest possible value of the cross section is $\pi T^{2}$. This limiting value decreases as the ion slows down as it approaches the end of its range, where the number of delta rays increases but their maximum energy decreases. Visually, we see the track end of a heavy ion in electron-sensitive emulsion looking like a sharpened pencil. This is called the region of "thin-down."

We note that in the thin-down region the cross section decreases as the stopping power increases. Such an inverse relation between cross section and stopping power is only found in the thin-down region, hence only in the track width regime. Thin-down has been observed with emulsion, scintillation counters, TLDs and for several radiobiological end points like cell killing, chromosome aberrations, and DNA strand breaks after bombardment with very heavy ions [8J.

Data dealing with particle tracks are frequently plotted against the stopping power $L$. A somewhat better plotting parameter is the ratio of the effective charge of an ion to its speed, $z^{*} / \beta$, or its square, for many effects are controlled by the number density of delta rays rather than by the soft collisions which contribute about 
half the energy loss and which vary at a somewhat different rate than delta ray production at different particle speeds. In the thin-down region neither of these parameters is particularly useful, and the use of $\beta$ as a plotting parameter may be helpful to identify thin-down, for $\sigma$ should decline toward $\mathrm{o}$ at the same rate for different heavy ions.

Thus we anticipate that in a plot of $\log \sigma$ vs $L$ (or vs $\left.\log \left(z^{*} / \beta\right)^{2}\right) \sigma$ increases nearly linearly with $L$ for 1 -hit detectors until the region of thin-down where it then declines. For 1-hit detectors, such a plot will yield no obvious clue as to the target size. For $C$-hit detectors, $(C \geq$ 2 ), the cross section increases approximately as $L^{C}$ in the grain count regime, passes through a plateau near the cross-sectional area of the target, and then increases linearly with $L$ in the track width regime up to the region of thin-down, where the cross section decreases. We find a marked branching of plots of $\sigma$ vs $L$ in the thin-down region at high stopping power for different bombarding ions. This is because in the thin-down region $\sigma$ is determined principally by the particle speed rather than the stopping power.

By introduction of sensitizers or desensitizers into a nuclear emulsion, its response to gamma rays can be markedly altered, with substantial changes in both $E_{\mathrm{o}}$ and $C$. These changes also affect the track appearance. Thus, in a sensitized emulsion the track of an argon ion may look like a hairy rope at all particle velocities and have a length of tens of $\mathrm{cm}$, in which the tracks of individual delta rays are observable. In a desensitized emulsion the track of an identical particle may only be observed for the stopping $100 \mu \mathrm{m}$ of its path, entirely in the grain count regime. At a greater range, when the particle has a higher velocity, the density of delta rays is too low for a sufficient number to pass through an emulsion grain and no individual delta rays are observable. Similar effects can be achieved by altering the $\mathrm{pH}$ or composition of the developer. In a parallel way the response of a biological system is altered by changes in pre- and post-irradiation culture conditions, and by a change in the genetic constitution of a cell which controls the production of repair enzymes. One must also expect the values of $E_{\mathrm{o}}$ and $C$ to alter with dopant composition and concentration in solid state detectors.

\section{Radiobiology}

Much radiobiological data does not lend itself to quantitative modelling, lacking in dynamic range in dose or LET or in temporal evolution to test a model. The data are frequently acquired for practical reasons related to radiation protection or therapy.

The radiobiological data best suited to quantitative model-building is obtained with simple systems like dry enzymes and viruses, bacterial spores, yeast, and mammalian cells, and using relatively simple tests like loss of function. We have already referred to the case of dry enzymes and viruses. These represent an ideal test system for any model. Enzyme or virus molecules are identical with each other, and have a specific biological test for loss of function. Further, their response to both gamma rays and heavy ions is measurable, and good statistics can be obtained. These are 1-hit detectors corresponding to loss of function of a molecule by its interaction with a single electron. As is typical of 1 -hit detectors, their relative biological effectiveness (RBE) declines with an increase in stopping power, also called linear energy transfer (LET), because of "overkill" near the ion's path. Though not yet observed, we have predicted the bombardments at which thin-down will be discovered.

A considerable body of existing radiobiological data suitable for quantitative models does exist in the form of cellular survival after irradiation with beams of energetic photons, electrons, and heavy ions from protons to uranium. The heavy ion data were initially obtained about 25 years ago using the HILAC accelerators at Yale and Berkeley, which yielded ions up to argon at energies up to about $10 \mathrm{MeV} / \mathrm{amu}$. More recent data for heavier and more energetic ions have been obtained at Berkeley and at Darmstadt.

Our analyses of these data follow the scheme developed for emulsions, with some modifications. First, the 3-parameter description of the characteristics of physical detectors is extended to a 4-parameter description of cells. As before, we avoid a mechanistic explanation of cell killing in favor of a global and parametric description. We imagine the targets in a mammalian cell to be within the the nuclear membrane, rather like beans in a bean bag, and that some number of these targets, $m$, must be inactivated in order to inactivate the cell (for cell killing really means loss of reproductive integrity). We imagine that there is an abundance of targets within the nucleus and that a heavy ion passing through a large portion of the nuclear volume may intersect and inactivate a suitable number of targets. Thus the variation of response with LET will depend on the size and properties of the bean, while the numerical value of the observed inactivation cross section will be related to the size of the bean bag.

We calculate the cross section for inactivation of a quasitarget (a single bean taken to represent the number of 1-hit beans which must be hit to inactivate the cell and whose radiosensitivity parameters are $E_{0}, a_{0}$, and $m$ ) for a wide range of parameter values. For these we find that in the grain count regime at low LET the cross section increases with LET as $L^{m}$ to a plateau value near the crosssectional area of the bean, while at high LET in the track width regime the cross section varies linearly with $L$ until we enter the region of thin-down where the cross sec- 
tion falls again. We approximate the envelope to these curves in the grain count regime by the expression

$$
P=\left[1-\exp \left(-z^{* 2} / \kappa \beta^{2}\right)\right]^{m},
$$

where $P$ is the probability for target inactivation and

$$
\kappa=E_{\mathrm{o}} a_{\mathrm{o}}^{2} /\left(2 \times 10^{-7} \mathrm{erg} / \mathrm{cm}\right) .
$$

We take $P$ to represent the ratio of the ion kill cross section $\sigma$ to its plateau value $\sigma_{0}$.

The fraction of intersected targets which is inactivated is $P$. These are inactivated in the ion kill mode. The fraction of ions which are intersected but not inactivated by a single ion is $(1-P)$. This residue may be inactivated in the gamma kill mode by intersecting delta rays from other ions. When a dose $D$ results from a heavy ion irradiation we take the dose fraction $P D$ to contribute to the ion kill mode of inactivation and the fraction $(1-P) D$ to contribute to the gamma kill mode of inactivation. We assume that the probability for ion kill follows 1-hit statistics, as in the first factor on the right in Equation (9), and that those cells surviving the ion kill mode may be inactivated in the gamma kill mode, as in the second factor on the right in Equation (9). Our expression for cellular survival after heavy ion bombardment is then

$$
N / N_{\mathrm{o}}=\exp (-\sigma F)\left\{1-\left[1-\exp \left(-(1-P) D / E_{\mathrm{o}}\right)\right]^{m}\right\}
$$

The equation includes four fitted parameters and gives the changing shape of survival curves with changes in the quantity $\left(z^{*} / \beta\right)^{2}$ of the bombarding ions. Once these parameters are evaluated we may extract the bean size $a_{\mathrm{o}}$ and recalculate the inactivation cross section for the particular cell line in the track width regime, and thus predict thin-down. With knowledge of the secondary particle energy spectrum produced by neutrons, or other high-LET irradiations, we can calculate the surviving fraction of cells irradiated in these modalities. This $4^{-}$ parameter equation has been fitted to the experimental data for the survival of a large variety of cells after heavyion irradiation as well as to cell transformation toward cancer induction, and chromosome damage. It has been used to describe heavy-ion damage to photoresists, with a presumed cluster of molecules taking the place of the cell nucleus [9].

\section{Discussion}

As presented here, we see that track theory provides a global model for track formation and the results of heavy-ion irradiation for a range of detectors, chemical, physical, and biological. The ideas are essentially the same in all cases, namely that the central contribution of atomic physics is knowledge of the average ra- dial distribution of dose about an ion's path. We neither need to know all the details of energy deposition, nor its fluctuation; and the central features of a detector are found from its response to gamma rays. These are clearly approximations. This model has been a remarkably successful one: it has accomplished its task, to describe the changing response of detectors with LET. It is the only global model of its kind; but we must remember that global models cannot be mechanistic, nor can mechanistic models be global. It has frequently been the case in physics that parametric fits to data have served to stimulate a mechanistic understanding, witness the relationship between the Balmer formula and the Bohr model of the hydrogen atom. Hopefully our present parametric model of particle tracks will serve to stimulate a mechanistic understanding of the behavior of the many detectors to which it has been applied, even to the mechanism through which ionizing radiations induce cancer.

This work was supported by the United States Department of Energy.

\section{References}

[1] R. Katz, S. C. Sharma, and M. Homayoonfar, in: Topics in Radiation Dosimetry, ed. F. H. Attix (Academic Press, New York, 1972) p. 317.

[2] R. Katz, Nucl. Track Detection 2 (1978) 1.

[3] R. Katz and E. J. Kobetich, Phys. Rev. 186 (1969) 344.

[4] R. Katz and GuoRong Huang, Radiation Physics and Chemistry (1989) in press.

[5] R. Katz, G. L. Sinclair, and M. P. R. Waligorski, Nucl. Tracks Radial. Meas. 11 (1986) 301.

[6] S. Z. Aghamohammadi, D. T. Goodhead, and J. R. K. Savage, Int. J. Radial. Biol., in press.

[7] M. P. R. Waligorski, K. S. Loh, and R. Katz, Radial. Phys. Chem. 30 (1987) 201.

[8] R. Katz, D. E. Dunn, and G. L. Sinclair, Radial. Prot. Dosimetry 13 (1985) 281.

[9] R. Katz, Proc. 8th Symp. in Microdosimetry, Commission of the European Communities (1983) eds. J. Booz and H. G. Ebert, p. 135 . 